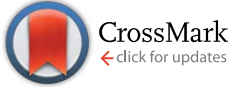

Cite this: Chem. Sci., 2015, 6, 2853

Received 24th January 2015

Accepted 26th February 2015

DOI: $10.1039 / \mathrm{c} 5 \mathrm{sc} 00273 \mathrm{~g}$

www.rsc.org/chemicalscience

\title{
Bis(dipyrrinato)metal(II) coordination polymers: crystallization, exfoliation into single wires, and electric conversion ability $\dagger$
}

\author{
Ryota Matsuoka, ${ }^{a}$ Ryojun Toyoda, ${ }^{a}$ Ryota Sakamoto, ${ }^{* a}$ Mizuho Tsuchiya, ${ }^{a}$ \\ Ken Hoshiko, ${ }^{a}$ Tatsuhiro Nagayama, ${ }^{a}$ Yoshiyuki Nonoguchi, ${ }^{b}$ Kunihisa Sugimoto, ${ }^{c}$ \\ Eiji Nishibori, ${ }^{d}$ Tsuyoshi Kawai ${ }^{\mathrm{b}}$ and Hiroshi Nishihara*a
}

\begin{abstract}
One-dimensional coordination polymers (1D-CPs) tend either to dissociate into constitutive ligands and metals readily in solution, or to aggregate randomly and amorphously, which prevents them from widespread application. In the present research, 1D-CPs comprising bridging dipyrrin ligands and divalent metal ions $\left(\mathrm{Zn}^{2+}, \mathrm{Ni}^{2+}\right.$, and $\left.\mathrm{Cu}^{2+}\right)$ are synthesized. A liquid/liquid interfacial reaction gives rise to single crystals suitable for $\mathrm{X}$-ray diffraction analysis: A dichloromethane solution of the ligand is layered with aqueous metal(॥) acetate, such that the coordination reaction proceeds at the liquid/liquid interface. Isolated single fibers of the zinc coordination polymer may be exfoliated from the single crystal or bulk solid upon ultrasonication. Atomic force microscopy (AFM) detects the isolated fibers with lengths of more than several $\mu \mathrm{m}$. The exfoliated $1 \mathrm{D}-\mathrm{CP}$ wires feature good processability, realizing a conjugate with single-wall carbon nanotubes (SWCNTs), and a thin film on a transparent $\mathrm{SnO}_{2}$ electrode. The processed materials show electric conversion ability: For example, the modified $\mathrm{SnO}_{2}$ electrode serves as a photoanode for a photoelectric conversion system. The designability and tunability of the present 1DCPs is demonstrated by a ligand modification, affording a luminescent property and an extension of the photoelectric conversion response to longer wavelengths.
\end{abstract}

\section{Introduction}

Two- and three-dimensional coordination polymers such as nanosheets, ${ }^{1-10}$ metal-organic frameworks (MOFs), ${ }^{11-13}$ and porous coordination polymers (PCPs) ${ }^{14-16}$ have attracted intense interest. On the other hand, the physical flexibility of onedimensional coordination polymers (1D-CPs) makes them

\footnotetext{
${ }^{a}$ Department of Chemistry, Graduate School of Science, The University of Tokyo, 7-3-1, Hongo, Bunkyo-ku, Tokyo 113-0033, Japan. E-mail: sakamoto@chem.s.u-tokyo.ac.jp; nisihara@chem.s.u-tokyo.ac.jp

${ }^{b}$ Graduate School of Materials Science, Nara Institute of Science and Technology (NAIST), 8916-5 Takayama, Ikoma, Nara 630-0192, Japan

'Japan Synchrotron Radiation Research Institute (JASRI), 1-1-1, Kouto, Sayo-cho, Sayogun, Hyogo 679-5198, Japan

${ }^{d}$ Division of Physics, Faculty of Pure and Applied Sciences, Tsukuba Research Center for Interdisciplinary Materials Science (TIMS), and Center for Integrated Research in Fundamental Science and Engineering (CiRfSE), University of Tsukuba, 1-1-1 Tennodai, Tsukuba, Ibaraki 305-8571, Japan

$\dagger$ Electronic supplementary information (ESI) available: Experimental methods; XPS of M1, Mono1, and Zn2; photographs of the single crystals of Ni1 and Cu1; single-crystal XRD data for M1; photograph of a free-standing film of Zn1-SWCNT; thermoelectric conversion of Zn1-SWCNT; photoelectric conversion and its quantitative analysis of Zn1, Zn2, and Mono3; photoelectric conversion setup. CCDC 1012353, 1044669 and 1044670. For ESI and crystallographic data in CIF or other electronic format See DOI: $10.1039 / \mathrm{c} 5 \mathrm{sc} 00273 \mathrm{~g}$
}

useful for conjugation with micro and nano-sized functional materials such as carbon nanotubes, ${ }^{17-19}$ although difficulties in handling have prevented 1D-CPs from widespread application. Many 1D coordination chains are stable only in the solid phase, and they dissociate into constitutive ligands and metals readily in solution. Furthermore, 1D-CPs tend to aggregate randomly and amorphously, and there are few examples of 1D-CPs where both crystalline phase and isolated single chain phase coexist. ${ }^{20,21}$

The dipyrrin-metal complex is an attractive molecular motif for coordination polymers. Dipyrrin ligands accept various metal ions, and in most cases the complexation reaction proceeds spontaneously even in the absence of a base. ${ }^{22,23}$ This feature is desirable for synthesizing supramolecules ${ }^{24,25}$ and coordination polymers. ${ }^{26-28}$ However, no 1D-CP based on the dipyrrin-metal complex has demonstrated crystallinity, singlechain isolation, and potential applicability at the same time.

In the present work, we synthesize 1D-CP M1 featuring the bis(dipyrrinato)metal(II) complex motif, which is composed of bridging dipyrrin ligand $\mathbf{L 1}$ and divalent metal ion $\mathbf{M}^{2+}(\mathbf{M}=\mathrm{Zn}$, $\mathrm{Ni}$, and $\mathrm{Cu}$, Fig. 1a). A liquid/liquid interfacial reaction is effective for ordering M1, giving rise to single crystals suitable for X-ray diffraction analysis (XRD). Single fibers of $\mathbf{Z n 1}$ are isolated from the single crystal upon ultrasonication, and then visualized by atomic force microscopy (AFM). The dispersibility 
a

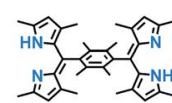

L1

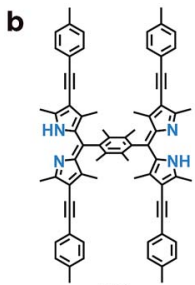

L2

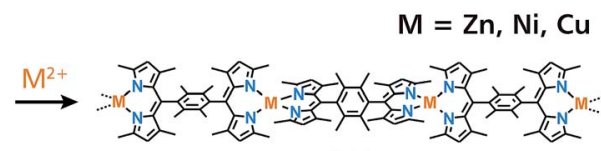

M1

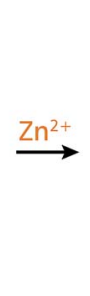

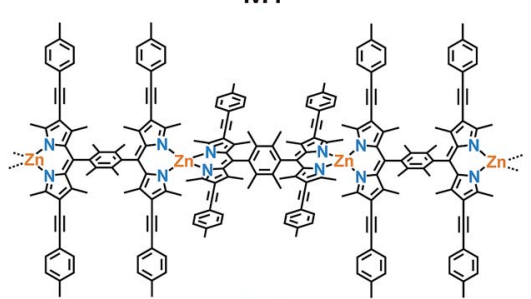

$\mathrm{Zn} 2$

Fig. 1 Chemical structures of bridging dipyrrin ligands and corresponding 1D-CPs based on bis(dipyrrinato)metal(॥) complexes. (a) L1 and $\mathrm{M} 1$ (M = Zn, Ni, Cu). (b) L2 and Zn2.

of the exfoliated fibers of $\mathbf{Z n 1}$ affords good processability, giving rise to a conjugate with single-wall carbon nanotubes (SWCNTs), and a thin film of $\mathbf{Z n 1}$ on a transparent $\mathrm{SnO}_{2}$ electrode. These processed materials may be applied in thermo and photoelectric conversion systems, thereby demonstrating the utility of Zn1. The designability and tunability of the present 1D$\mathrm{CP}$ system are illustrated with analogue $\mathbf{Z n 2}$ comprising $\pi$ extended ligand L2 (Fig. 1b), which shows luminescence in the exfoliated fibrous form, and an extension of the photoelectric conversion response to longer wavelengths.

\section{Results and discussion}

Initially, a conventional single-phase reaction was performed to synthesize Zn1 (Fig. 1a). Equimolar amounts of bridging dipyrrin ligand $\mathbf{L 1}$ and zinc(II) acetate were reacted in a mixture of dichloromethane and ethanol (Fig. 2a and b). The resultant dark-orange powder was subjected to X-ray photoelectron spectroscopy (XPS) using L1 and mononuclear bis(dipyrrinato) zinc(II) complex Mono1 as references (Fig. 2c). The Zn 2p 3/2 peak is only visible in Mono1 and Zn1, which is consistent with the presence of $\mathrm{Zn}$ ions. Another distinctive fingerprint of the coordination between the dipyrrin ligand and zinc(II) ions occurs in the $\mathrm{N} 1 \mathrm{~s}$ region. Free base $\mathbf{L 1}$ features two peaks (397.5 and $399.1 \mathrm{eV}$ ) arising from the iminic and pyrrolic nitrogens that are chemically different from one another. ${ }^{29}$ In contrast, Mono1 shows a single N 1s peak, which stems from the homogenization of the two nitrogen atoms upon coordination to the zinc center. A solitary $\mathrm{N} 1 \mathrm{~s}$ peak is also observed for Zn1. In addition, the nitrogen-to-zinc abundance ratio calculated from the peak area corrected by the photoionization cross-section is consistent with the ideal value of $\mathrm{N}: \mathrm{Zn}=4: 1$ (79.8: 20.2 and $79.5: 20.5$ for $\mathbf{Z n 1}$ and Mono1, respectively, Fig. S1a and $\mathrm{b} \dagger)$. These results indicate the formation of the desired coordination polymer Zn1 consisting of the bis(dipyrrinato)zinc(II) complex. The authors note that Ni1 and Cu1 synthesized by means of the single-phase method also displayed nitrogen-to-metal abundance ratios of $c a .4: 1$ in XPS (80.2 : 19.8 and $80.3: 19.7$ for Ni1 and Cu1, respectively, Fig. S1c and $d \dagger$ ).

a
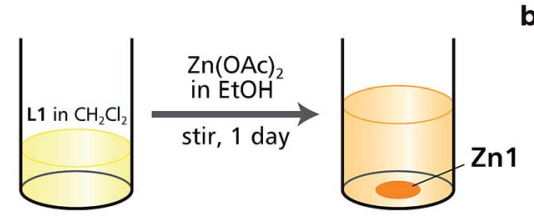

b

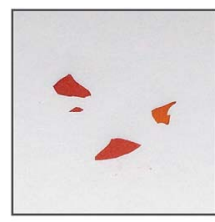

c
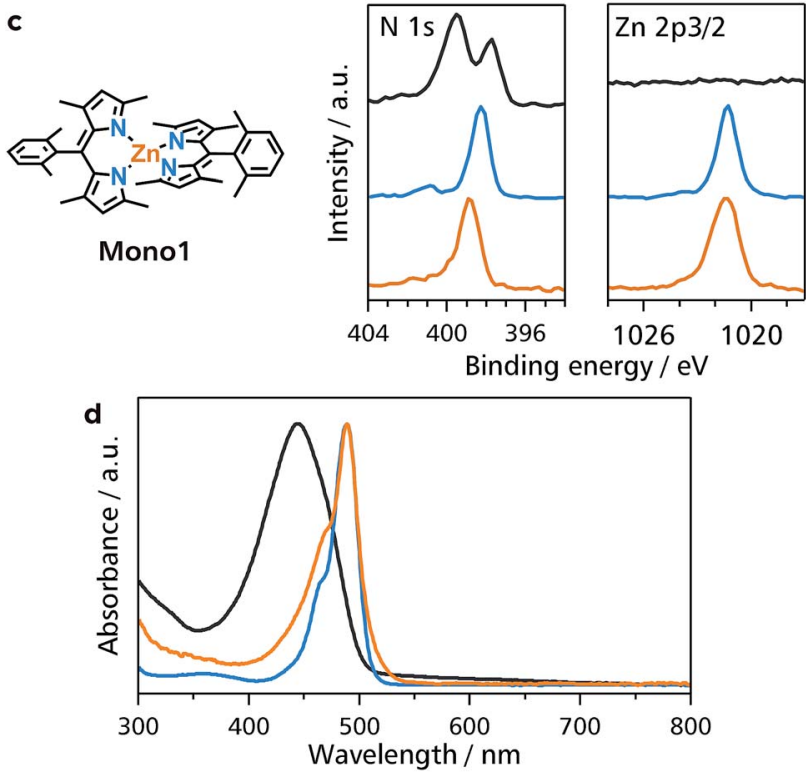

Fig. 2 Single-phase synthesis and characterization of Zn1. (a) Schematic illustration of the synthesis. (b) Photograph of powdery Zn1. (c) Chemical structure of referential compound Mono1, and XPS of L1 (black), Mono1 (blue), and Zn1 (orange) focusing on the N 1s and Zn 2p $3 / 2$ regions. (d) UV/vis spectra of $\mathrm{L} 1$ (black), Mono1 (blue), and Zn1 (orange) in DMF.

Zn1 is dispersible in dimethylformamide, and was characterized by UV/vis spectroscopy (Fig. 2d). An intense absorption with a maximum at $445 \mathrm{~nm}$ in $\mathbf{L} 1$ stems from the ${ }^{1} \pi-\pi^{*}$ transition of the free-base dipyrrin moiety. ${ }^{30}$ However, the ${ }^{1} \pi-\pi^{*}$ band is red-shifted by $44 \mathrm{~nm}$ in Mono1 (489 nm), which is typical of zinc(II) complexation with a dipyrrin ligand. ${ }^{30}$ The $^{1} \pi-$ $\pi^{*}$ band of $\mathbf{Z n 1}$ has the same absorption maximum ( $489 \mathrm{~nm}$ ) as that of Mono1, which also shows that Zn1 formed the bis(dipyrrinato)zinc(II) coordination polymer.

We have demonstrated previously that liquid/liquid interfacial synthesis-where organic ligand molecules in an organic solvent and aqueous metal ions are layered to form a liquid/ liquid interface-is effective for synthesizing low-dimensional CPs with ordered secondary structures. ${ }^{4,5,31,32}$ For example, a liquid/liquid interfacial synthesis using 1,2,4,5-benzenetetrathiol and nickel(II) ions produced a nickel bis(dithiolene) 1D$\mathrm{CP}$ that aligns to form two-dimensional ordered structures. ${ }^{31} \mathrm{In}$ the present work, the coordination reaction between bridging dipyrrin ligand $\mathbf{L} 1$ in dichloromethane and divalent metal ions $\mathbf{M}^{2+}(\mathbf{M}=\mathrm{Zn}, \mathrm{Ni}$, and $\mathrm{Cu})$ in water was carried out at the liquid/ liquid interface, and it produced crystals of M1 that floated on the interface or sank to the bottom of the reaction container (Fig. 3a and b). A photograph of a typical single crystal for $\mathbf{Z n} \mathbf{1}$ is shown in Fig. 3c, and those for Ni1 and $\mathbf{C u 1}$ are placed in 
a

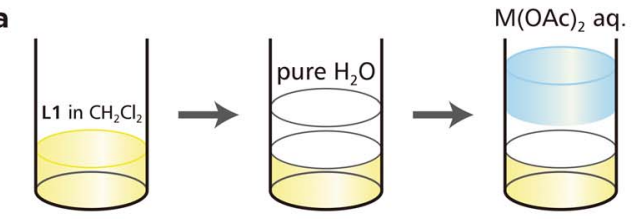

b

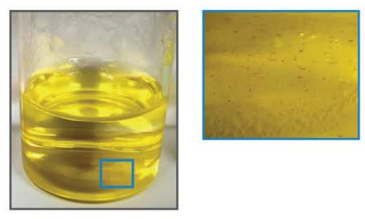

c

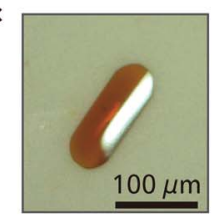

d

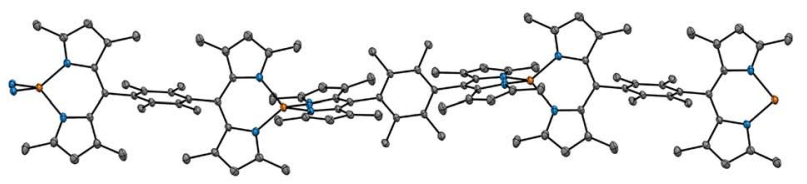

e
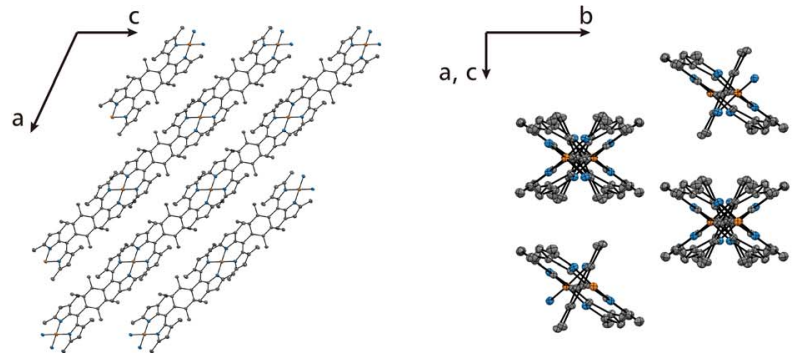

Fig. 3 Liquid/liquid interfacial synthesis for M1 and crystal structure of Zn1. (a) Schematic illustration of the liquid/liquid interfacial synthesis for M1. (b) (left) Photograph of the reaction system after the emergence of single crystals of Zn1 and (right) close-up image at the liquid/liquid interface (blue square). (c) Photograph of a typical single crystal of Zn1. (d) ORTEP drawing of Zn1 with a thermal ellipsoid set at the 50\% probability level (C, gray; N, blue; Zn, orange). Hydrogen atoms are omitted for clarity. (e) Perspective views of 3D packing of Zn1 along the b (left) and [1 $0-1]$ (right) crystallographic axes, respectively.

Fig. S2. $\dagger$ This series of crystals was analyzed by synchrotron radiation X-ray diffraction (Fig. $3 \mathrm{~d}$ and e and $\mathrm{S} 3, \dagger$ and Table $\mathrm{S} 1 \dagger$ for Zn1, Fig. S3 and Table S2† for Ni1, and Fig. S3 and Table S3† for Cu1)..$^{33}$ The crystal structures are almost identical to each other for the three metal centers. They show the desired 1D polymeric chains, propagating along the $\left[\begin{array}{lll}1 & 0 & -1\end{array}\right]$ crystallographic axis. The metal centers adopt slightly distorted tetrahedral coordination spheres with dihedral angles of $81.29^{\circ}$, $81.11^{\circ}$, and $87.36^{\circ}$ for $\mathrm{Zn}, \mathrm{Ni}$, and $\mathrm{Cu}$, respectively. The average metal-nitrogen bond lengths are, respectively, 1.9181, 1.9151, and $1.9032 \AA$ for $\mathrm{Zn}, \mathrm{Ni}$, and $\mathrm{Cu}$. This series of dihedral angles and metal-nitrogen bond lengths are typical of bis(dipyrrinato) metal(II) complexes bearing $\alpha$-methylated dipyrrin ligands..$^{30,34}$ Divalent nickel and copper generally prefer square-planar coordination spheres, though, the methyl group at the $\alpha$-position induces tetrahedral coordination spheres even to the $\mathrm{Ni}$ and $\mathrm{Cu}$ centers because of steric hindrance. The dipyrrinato ligand is orthogonal to the bridging 2,3,4,5-tetramethylphenyl group with dihedral angles of $101.18^{\circ}, 100.02^{\circ}$, and $98.93^{\circ}$ for $\mathrm{Zn}, \mathrm{Ni}$, and $\mathrm{Cu}$, respectively, reflecting steric hindrance between the two moieties.

Upon ultrasonication, the single crystal of Zn1 could be dispersed in dichloromethane, and showed Tyndall scattering (Fig. 4a). The dispersion was cast onto highly ordered pyrolytic graphite (HOPG), which was then subjected to AFM. A representative height image shows several straight white lines with lengths of $>3 \mu \mathrm{m}$ traversing the steps of the HOPG substrate (Fig. 4b). The cross-sectional analysis shows that the height of the lines is $c a .0 .7 \mathrm{~nm}$ (Fig. 4c), which is consistent with the size of Mono1 estimated by means of DFT calculation (Fig. 4d). This indicates that Zn1 is stable enough both chemically and physically to be isolated as single chains. We note that the width of Zn1 is overestimated by AFM. This is common in 1D polymer systems and AFM resolution. ${ }^{35-37}$

Among bis(dipyrrinato)metal(II) complexes, zinc-centered ones are known to possess luminescence ability. Nevertheless, Zn1 did not show detectable fluorescence at room temperature.
This situation was improved by one of the virtues of 1D-CPs, designability and tunability: The authors also synthesized $\pi$ extended bridging dipyrrin ligand $\mathbf{L 2}$, and prepared another 1D-CP Zn2 (Fig. 1b). In XPS, Zn2 showed a nitrogen-to-metal abundance ratio of $79.4: 20.6$, almost consistent with the ideal value ( $4: 1)$ (Fig. S1e $\dagger)$. Thanks to the (4-methylphenyl)ethynyl group at the $\beta$-position of the dipyrrin ligand, the dispersibility of $\mathbf{Z n} 2$ was better than that of $\mathbf{Z n 1}$. In addition, Zn2 featured orange emission in a dichloromethane dispersion, so that Tyndall scattering was concealed (Fig. 5a). In UV/vis and luminescence spectroscopy in toluene (Fig. 5b), Zn2 featured the ${ }^{1} \pi-\pi^{*}$ absorption with a maximum at $547 \mathrm{~nm}$, which is similar to that of corresponding mononuclear complex Mono2 $\left(\lambda_{\max }=553 \mathrm{~nm}\right.$, Fig. $5 \mathrm{~b}$ and $\left.\mathrm{c}\right)$. These bands are red-shifted relative to that of $\mathbf{L} 2\left(\lambda_{\max }=505 \mathrm{~nm}\right)$, indicative of the a

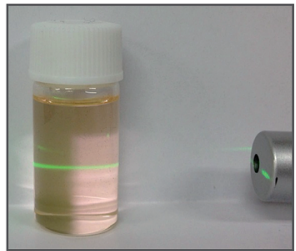

b

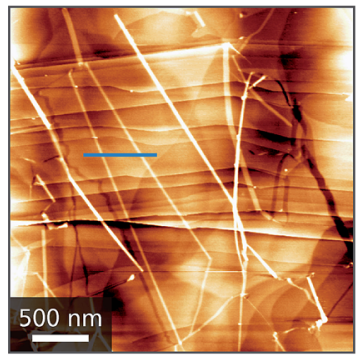

d

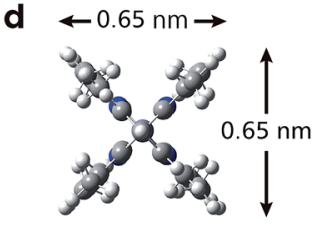

Fig. 4 Exfoliation of single fibers of Zn1. (a) Tyndall scattering from a dichloromethane colloidal suspension of $Z n 1$ upon illumination with a green luminous flux. (b) AFM topographic image of exfoliated fibers of Zn1 on HOPG. (c) AFM cross-sectional profile along the blue line in (b). (d) Molecular size of Mono1 estimated by means of DFT calculation. 
complexation with zinc(II) ions. In contrast to non-fluorescent Zn1, the fluorescence of Zn2 was observed at the maximum wavelength of $597 \mathrm{~nm}$ (quantum efficiency: 8\%): therefore, the fluorescence ability revived upon the ligand modification in $\mathbf{L 2}$. AFM for a HOPG substrate modified with a dispersion of Zn2 disclosed straight white lines with a length of more than $4.5 \mu \mathrm{m}$ (Fig. 5d and e). The height of the lines $(1.9 \mathrm{~nm})$ is in good agreement with the size of Mono2 estimated by DFT calculation (Fig. 5f).

In order to demonstrate the processability of $\mathbf{Z n 1}$, the authors fabricated a conjugate with SWCNTs (Zn1-SWCNT). A mixture of Zn1 and SWCNTs with a weight ratio of $1: 10$ was dispersed in DMF by ultrasonication for $90 \mathrm{~min}$, and the dispersion was then shaken for $1 \mathrm{~d}$. This process resulted in the
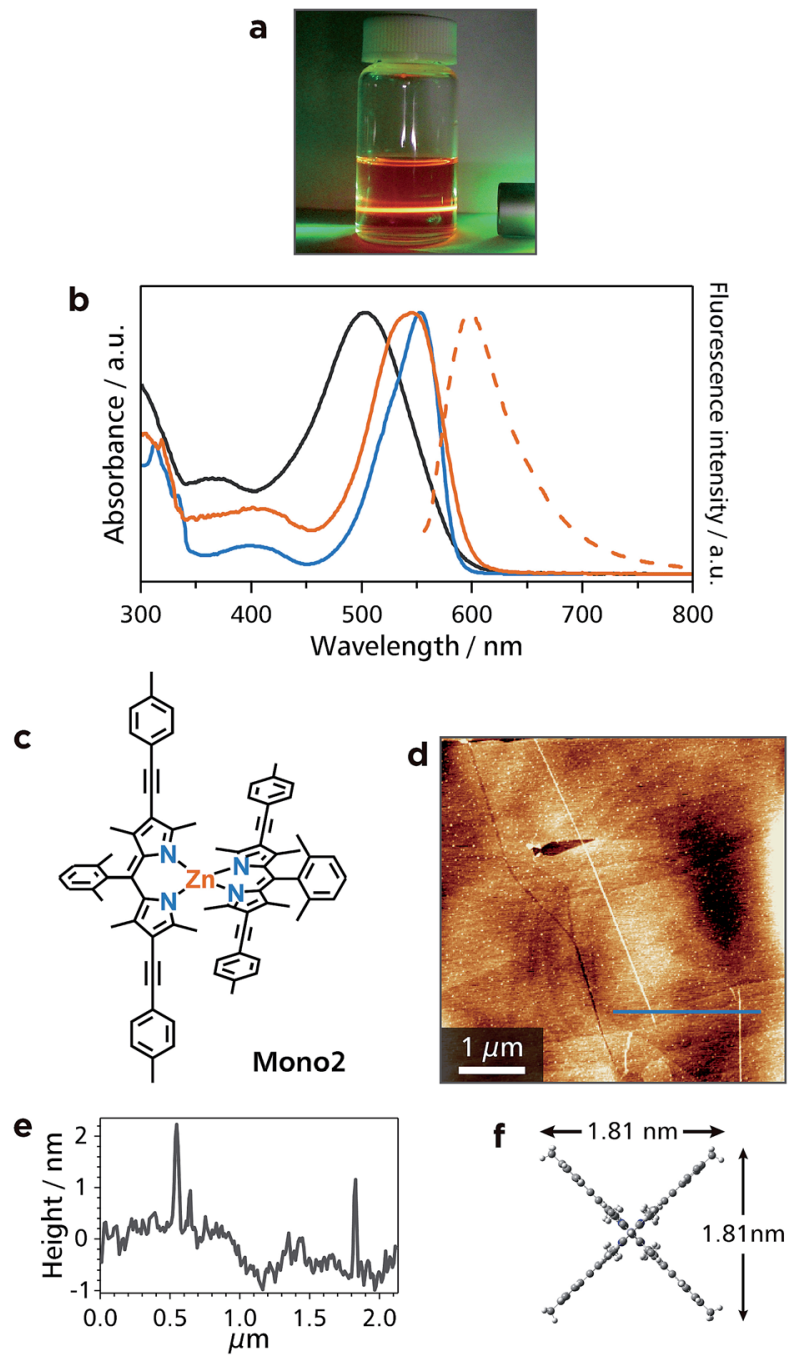

Fig. 5 Characterization of Zn2. (a) Dichloromethane dispersion of Zn2 irradiated with a green laser. Tyndal scattering was concealed by orange fluorescence from Zn2. (b) Absorption (solid lines) and fluorescence (dotted line) spectra of L2 (black), Mono2 (blue), and Zn2 (orange) in toluene. (c) Chemical structure of referential mononuclear zinc(II) complex Mono2. (d) AFM topographic image of HOPG modified with a dispersion of Zn2. (e) AFM cross-sectional profile along the blue line in (d). (f) Molecular size of Mono2 estimated by means of DFT calculation. disappearance of the orange color of $\mathbf{Z n 1}$ from DMF, which indicated that Zn1 was adsorbed onto SWCNTs. Upon filtration, the conjugate spontaneously assembled into a free-standing membrane with a thickness of $64 \mu \mathrm{m}$ (Fig. S4 $\dagger$ ). The conjugate was then subjected to spectroscopic analyses. Fig. 6a-c shows TEM images of bundles of SWCNTs with some exfoliated tubes. On the other hand, electron energy-loss spectroscopy (EELS) reveals the presence of zinc, which is scattered uniformly across the carbon scaffold derived from the SWCNT skeleton (Fig. 6dg). Raman spectroscopy shows an intense $\mathrm{G}$ band $\left(1590 \mathrm{~cm}^{-1}\right)$ and a weak D band $\left(1350 \mathrm{~cm}^{-1}\right)$, which stem from intact and damaged SWCNTs, respectively (Fig. 6h). ${ }^{38}$ These results indicate that Zn1 wraps around the SWCNT uniformly without destroying the SWCNT structure. A preliminary experiment disclosed the thermoelectric conversion ability of Zn1-SWCNT (Fig. S5 $\dagger$ ). Zn1-SWCNT has a power factor of $33 \mu \mathrm{W} \mathrm{m}{ }^{-1} \mathrm{~K}^{-2}$, which is greater than that of pristine SWCNTs $\left(9.3 \mu \mathrm{W} \mathrm{m}{ }^{-1}\right.$ $\mathrm{K}^{-2}$ ), and those derived from conjugates between SWCNTs and small organic molecules $\left(26 \mu \mathrm{W} \mathrm{m}{ }^{-1} \mathrm{~K}^{-2}\right.$ at most $){ }^{39}$

Thanks to intense light absorption disclosed in Fig. 2d and 5b, Zn1 and $\mathbf{Z n 2}$ are expected to show photofunctionality. As one of such demonstrations, $\mathbf{Z n 1}$ and $\mathbf{Z n 2}$ were built into a photoelectric conversion system. Dispersions of Zn1 and Zn2 were deposited onto transparent $\mathrm{SnO}_{2}$ electrodes, such that thin films of Zn1 and Zn2 were formed (Fig. 7a and S6a $\dagger$ ): This also ensures the good processability of $\mathbf{Z n 1}$ and $\mathbf{Z n 2}$. A three-electrode electrochemical cell was then set up (Fig. S7†), where the modified $\mathrm{SnO}_{2}$ electrode was employed as a photoanode. An
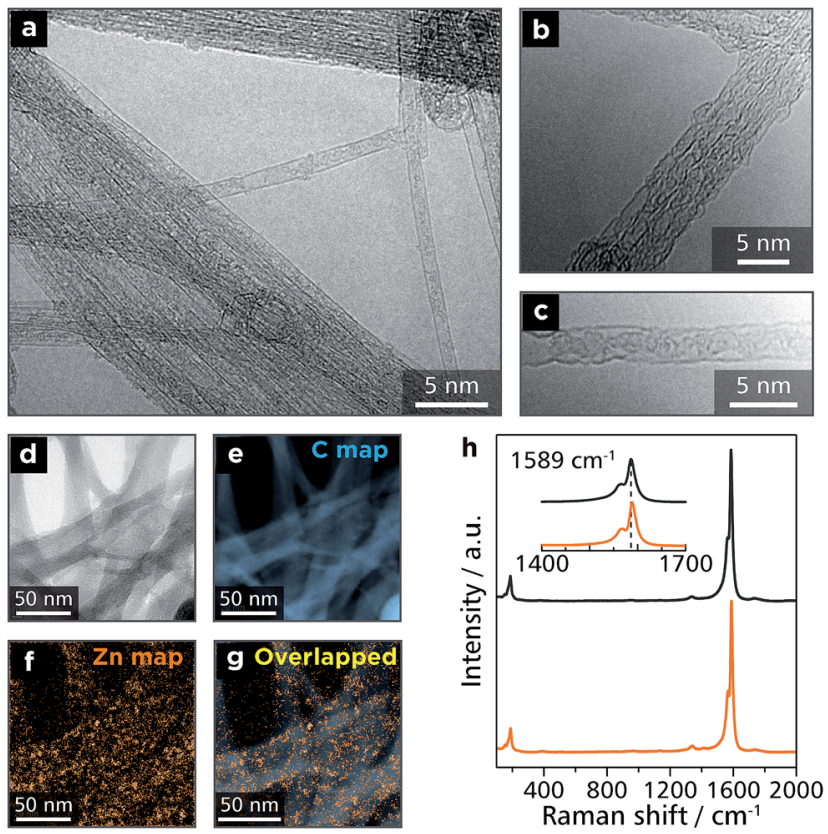

Fig. 6 Microscopic and spectroscopic analyses for Zn1-SWCNT. (a)(c) Transmission electron microscopy (TEM) images of Zn1-SWCNT on a Cu grid. (d) Bright-field-TEM image of Zn1-SWCNT subjected to electron energy-loss spectroscopy (EELS) mapping. EELS mapping for: (e) carbon $\mathrm{K}$ edge intensity and ( $\mathrm{f}$ ) zinc $\mathrm{M} 2$ and $\mathrm{M} 3$ edge intensities. (g) Overlapped image of (e) and (f). (h) Raman spectra of pristine SWCNTs (black) and Zn1-SWCNT (orange). 
a

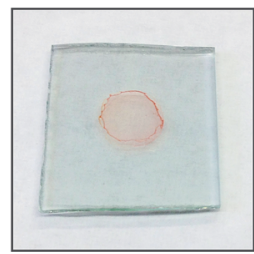

b

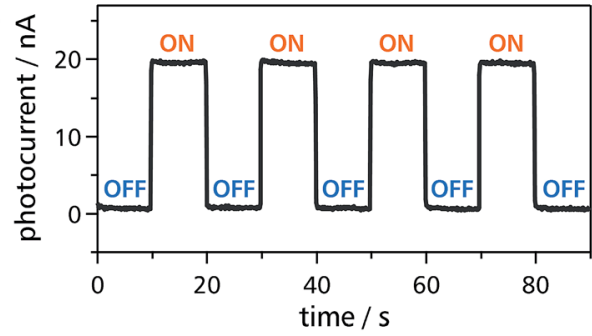

c

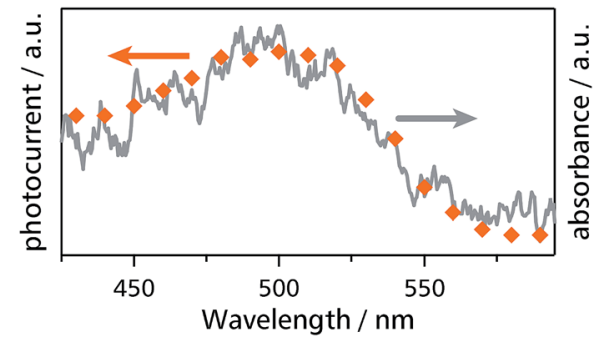

Fig. 7 Photoelectric conversion employing $\mathrm{Zn} 1$ as an active material. (a) Photograph of a thin film of $\mathrm{Zn1}$ on a $\mathrm{SnO}_{2}$ electrode. (b) Typical anodic photocurrent response upon irradiation of a working electrode $\left(\mathrm{SnO}_{2}\right.$ substrate modified with $\mathrm{Zn1}$ as shown in (a)) with intermittent $500 \mathrm{~nm}$ light. (c) Action spectrum for the photocurrent generation (orange dots) and absorption spectrum of $\mathrm{Zn1}$ on a $\mathrm{SnO}_{2}$ substrate (gray solid line).

anodic photocurrent was observed only when the Zn1-modified $\mathrm{SnO}_{2}$ electrode was illuminated with $500 \mathrm{~nm}$ light (Fig. 7b). In addition, the action spectrum for the photocurrent coincided with the absorption spectrum of $\mathbf{Z n 1}$ on a $\mathrm{SnO}_{2}$ electrode (Fig. 7c). This series of facts indicates that Zn1 functions as an active layer for the photoelectric conversion system. The authors then investigated the photoelectric conversion efficiency (Fig. S8 and S9 $\uparrow$ ). As shown in Fig. S9a, $\dagger$ the photocurrent reaches the maximal value with the optical density of the film at $500 \mathrm{~nm}$ of $\sim 0.005$. On the other hand, the quantum yield for the photoelectric conversion decreased as the growth of the film, showing the maximal value of $1.0 \%$ in an acetonitrile medium (Fig. S9b†). To demonstrate the predominance of $\mathbf{Z n 1}$, the authors also prepared mononuclear bis(dipyrrinato)zinc(II) complex Mono3 ${ }^{32}$ with carboxy groups, which forms a selfassembled monolayer (SAM) on a $\mathrm{SnO}_{2}$ electrode upon chemisorption (Fig. S10a $\dagger$ ). The SAM of Mono3 performed a conversion efficiency of $0.069 \%$ in an acetonitrile medium (Fig. S10b and $c^{\dagger}$ ), which is much lower than that of $\mathbf{Z n 1}$ with the same optical density. The $\mathbf{Z n 2 - m o d i f i e d ~} \mathrm{SnO}_{2}$ electrode also served as a photoanode in the same manner as $\mathbf{Z n 1}$, except that the photoresponse is red-shifted, which is esteemed in photoelectric conversion applications (Fig. S6b and $c^{\dagger}$ ). The red-shift is induced by the $\pi$-extension of dipyrrin ligand $\mathbf{L} 2$, thereby demonstrating the designability and tunability of the present
1D-CPs. The highest conversion efficiency of $\mathbf{Z n 2}(0.027 \%$ in an aqueous medium, Fig. S6d and $\mathrm{S} 11 \dagger)$ is far greater than that of Mono3 in the same medium ( $\sim 0 \%$, Fig. S10d and e $\dagger$ ). The series of comparative experiments displays that the polymer structure of $\mathbf{Z n 1}$ and $\mathbf{Z n 2}$ is advantageous for photoelectric conversion applications.

\section{Conclusions}

The authors determined the structural and functional features of 1D-CPs comprising the bis(dipyrrinato)metal(II) complex motif. Layering bridging dipyrrin ligand $\mathbf{L 1}$ in dichloromethane and metal(II) ions in water produced single crystals of 1D-CP M1 through a spontaneous coordination reaction at the liquid/ liquid interface. X-ray diffraction analysis showed that the fibers

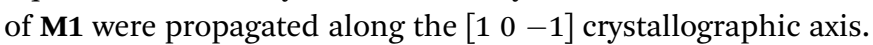
Isolated fibers of $\mathbf{Z n} \mathbf{1}$ could be exfoliated from the single crystal upon ultrasonication in dichloromethane, and AFM confirmed that the fibers were more than several $\mu \mathrm{m}$ long. Zn1 did not show detectable fluorescence, whereas 1D-CPs Zn2 comprising $\pi$-extended bridging dipyrrin ligand $\mathbf{L} 2$ and zinc(II) ions featured orange fluorescence with the maximum wavelength of $597 \mathrm{~nm}$ in a toluene dispersion: this demonstrated the designability and tunability of the present 1D-CP. The dispersibility of Zn1 and Zn2 was sufficient enough to afford a significant advantage in processing them for applications. Zn1 formed a conjugate with SWCNTs, where the fibers of Zn1 wrapped around SWCNTs uniformly, and which featured thermoelectric conversion. In addition, $\mathbf{Z n} 1$ and $\mathbf{Z n} 2$ could be deposited onto transparent $\mathrm{SnO}_{2}$ electrodes as thin films, which served as photoanodes for a photoelectric conversion system. The photoelectric conversion response could be extended to longer wavelengths by a dipyrrin ligand modification. Our present results herein highlight the utility of $1 \mathrm{D}$-CPs in materials science.

\section{Acknowledgements}

The authors acknowledge Grants-in-Aid from MEXT of Japan (nos 21108002, 24750054, 25107510, 26708005, 26107510, 26620039, 26220801, 26248017, 26110505, areas 2107 [Coordination Programming], 2406 [All Nippon Artificial Photosynthesis Project for Living Earth], 2506 [Science of Atomic Layers], 2509 [Molecular Architectonics]), and JSPS fellowship for young scientists. R.S. is grateful to The Japan Prize Foundation, Iketani Science and Technology Foundation, The Murata Science Foundation, Tokuyama Science Foundation, Ogasawara Foundation for the Promotion of Science \& Engineering, The Kao Foundation for Arts and Sciences, The Asahi Glass Foundation, The Noguchi Institute, Japan Association for Chemical Innovation, The MIKIYA Science and Technology Foundation, Yazaki Memorial Foundation for Science and Technology, Shorai Foundation for Science and Technology, The Kurata Memorial Hitachi Science and Technology Foundation, and Kumagai Foundation for Science and Technology for financial supports. The synchrotron radiation experiment was performed at the BL02B1 (ref. 40) of SPring-8 (Proposal no. 2014A1355) 
with the approval of the Japan Synchrotron Radiation Research Institute (JASRI). The authors acknowledge the Research Hub Advanced Nano Characterization (Graduate School of Engineering, The University of Tokyo) for the XPS study.

\section{Notes and references}

1 D. Sheberla, L. Sun, M. A. Blood-Forsythe, S. Er, C. R. Wade, C. K. Brozek, A. Aspuru-Guzik and M. Dincă, J. Am. Chem. Soc., 2014, 136, 8859-8862.

2 J. Cui and Z. Xu, Chem. Commun., 2014, 50, 3986-3988.

3 G. Xu, K. Otsubo, T. Yamada, S. Sakaida and H. Kitagawa, J. Am. Chem. Soc., 2013, 135, 7438-7441.

4 T. Kambe, R. Sakamoto, T. Kusamoto, T. Pal, N. Fukui, T. Shimojima, Z. Wang, T. Hirahara, K. Ishizaka, S. Hasegawa, F. Liu and H. Nishihara, J. Am. Chem. Soc., 2014, 136, 14357-14360.

5 T. Kambe, R. Sakamoto, K. Hoshiko, K. Takada, J.-H. Ryu, S. Sasaki, J. Kim, K. Nakazato, M. Takata and H. Nishihara, J. Am. Chem. Soc., 2013, 135, 2462-2465.

6 H. Nishihara, Chem. Lett., 2014, 43, 388-395.

7 P. Amo-Ochoa, L. Welte, R. González-Prieto, P. J. S. Miguel, C. J. Gómez-García, E. Mateo-Martí, S. Delgado, J. GómezHerrero and F. Zamora, Chem. Commun., 2010, 46, 32623264 .

8 J.-C. Tan, P. J. Saines, E. G. Bithell and A. K. Cheetham, ACS Nano, 2012, 6, 615-621.

9 T. Bauer, Z. Zheng, A. Renn, R. Enning, A. Stemmer, J. Sakamoto and A. D. Schlüter, Angew. Chem., Int. Ed., 2011, 50, 7879-7884.

10 Z. Zheng, L. Opilik, F. Schiffmann, W. Liu, G. Bergamini, P. Ceroni, L.-T. Lee, A. Schütz, J. Sakamoto, R. Zenobi, J. VandeVondele and A. D. Schlüter, J. Am. Chem. Soc., 2014, 136, 6103-6110.

11 H. Furukawa, K. E. Cordova, M. O'Keeffe and O. M. Yaghi, Science, 2013, 341, 1230444.

12 M. Sadakiyo, T. Yamada, K. Honda, H. Matsui and H. Kitagawa, J. Am. Chem. Soc., 2014, 136, 7701-7707.

13 T. Li, M. T. Kozlowski, E. a. Doud, M. N. Blakely and N. L. Rosi, J. Am. Chem. Soc., 2013, 135, 11688-11691.

14 R. Matsuda, R. Kitaura, S. Kitagawa, Y. Kubota, R. V Belosludov, T. C. Kobayashi, H. Sakamoto, T. Chiba, M. Takata and Y. Kawazoe, Nature, 2005, 436, 238-241.

15 H. Deng, S. Grunder, K. E. Cordova, C. Valente, H. Furukawa, M. Hmadeh, F. Gándara, A. C. Whalley, Z. Liu and S. Asahina, Science, 2012, 336, 1018-1023.

16 Y. Inokuma, S. Yoshioka, J. Ariyoshi, T. Arai, Y. Hitora, K. Takada, S. Matsunaga, K. Rissanen and M. Fujita, Nature, 2013, 495, 461-466.

17 L. Ren, L. Yang, P. Yu, Y. Wang and L. Mao, ACS Appl. Mater. Interfaces, 2013, 5, 11471-11478.

18 J. T. Culp, L. Sui, A. Goodman and D. Luebke, J. Colloid Interface Sci., 2013, 393, 278-285.
19 J. Liu, M. Chen, D.-J. Qian and M. Liu, RSC Adv., 2014, 4, 5678-5682.

20 D. Olea, S. S. Alexandre, P. Amo-Ochoa, A. Guijarro, F. de Jesús, J. M. Soler, P. J. de Pablo, F. Zamora and J. GómezHerrero, Adv. Mater., 2005, 17, 1761-1765.

21 U. García-Couceiro, D. Olea, O. Castillo, A. Luque, P. Román, P. J. de Pablo, J. Gómez-Herrero and F. Zamora, Inorg. Chem., 2005, 44, 8343-8348.

22 T. E. Wood and A. Thompson, Chem. Rev., 2007, 107, 18311861.

23 S. Baudron, Dalton Trans., 2013, 42, 7498-7509.

24 H. Maeda and T. Hashimoto, Chem.-Eur. J., 2007, 13, 79007907.

25 H. Maeda, T. Nishimura, R. Akuta, K. Takaishi, M. Uchiyama and A. Muranaka, Chem. Sci., 2013, 4, 1204-1211.

26 H. Maeda, M. Hasegawa, T. Hashimoto, T. Kakimoto, S. Nishio and T. Nakanishi, J. Am. Chem. Soc., 2006, 128, 10024-10025.

27 A. Béziau, S. Baudron, A. Guenet and M. W. Hosseini, Chem.Eur. J., 2013, 19, 3215-3223.

28 B. Kilduff, D. Pogozhev, S. Baudron and M. W. Hosseini, Inorg. Chem., 2010, 49, 11231-11239.

29 D. H. Karweik and N. Winograd, Inorg. Chem., 1976, 15, 2336-2342.

30 S. Kusaka, R. Sakamoto, Y. Kitagawa, M. Okumura and H. Nishihara, Chem.-Asian J., 2012, 7, 907-910.

31 R. Matsuoka, R. Sakamoto, T. Kambe, K. Takada, T. Kusamoto and H. Nishihara, Chem. Commun., 2014, 50, 8137-8139.

32 R. Sakamoto, K. Hoshiko, Q. Liu, T. Yagi, T. Nagayama, S. Kusaka, M. Tsuchiya, Y. Kitagawa, W.-Y. Wong and H. Nishihara, Nat. Commun., 2015, 6, 6713.

$33 \mathrm{ESI}^{\dagger}$

34 Q. Miao, J.-Y. Shin, B. O. Patrick and D. Dolphin, Chem. Commun., 2009, 2541-2543.

35 D. Olea, U. García-Couceiro, O. Castillo, J. Gómez-Herrero and F. Zamora, Inorg. Chim. Acta, 2007, 360, 48-54.

36 P. Amo-Ochoa, M. I. Rodríguez-Tapiador, O. Castillo, D. Olea, A. Guijarro, S. S. Alexandre, J. Gómez-Herrero and F. Zamora, Inorg. Chem., 2006, 45, 7642-7650.

37 L. Welte, R. González-Prieto, D. Olea, M. R. Torres, J. L. Priego, R. Jiménez-Aparicio, J. Gómez-Herrero and F. Zamora, ACS Nano, 2008, 2, 2051-2056.

38 M. S. Dresselhaus, G. Dresselhaus, R. Saito and A. Jorio, Phys. Rep., 2005, 409, 47-99.

39 Y. Nonoguchi, K. Ohashi, R. Kanazawa, K. Ashiba, K. Hata, T. Nakagawa, C. Adachi, T. Tanase and T. Kawai, Sci. Rep., 2013, 3, 3344 .

40 K. Sugimoto, H. Ohsumi, S. Aoyagi, E. Nishibori, C. Moriyoshi, Y. Kuroiwa, H. Sawa and M. Takata, AIP Conf. Proc., 2010, 1234, 887-890. 